\title{
Targeting the HA2 subunit of influenza A virus hemagglutinin via CD40L provides universal protection against diverse subtypes
}

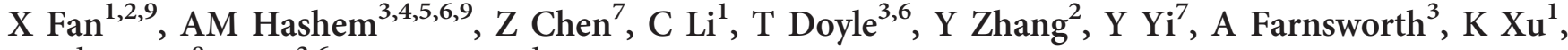 \\ $\mathrm{Z} \mathrm{Li}^{1}, \mathrm{R} \mathrm{He}^{8}, \mathrm{X} \mathrm{Li}^{3,6}$ and $\mathrm{J}$ Wang ${ }^{1}$
}

The influenza viral hemagglutinin $(\mathrm{HA})$ is comprised of two subunits. Current influenza vaccine predominantly induces neutralizing antibodies (Abs) against the HA1 subunit, which is constantly evolving in unpredictable fashion. The other subunit, HA2, however, is highly conserved but largely shielded by the HA head domain. Thus, enhancing immune response against HA2 could potentially elicit broadly inhibitory Abs. We generated a recombinant adenovirus ( $\mathrm{rAd}$ ) encoding secreted fusion protein, consisting of codon-optimized HA2 subunit of influenza A/California/7/2009(H1N1) virus fused to a trimerized form of murine CD40L, and determined its ability of inducing protective immunity upon intranasal administration. We found that mice immunized with this recombinant viral vaccine were completely protected against lethal challenge with divergent influenza A virus subtypes including H1N1, H3N2, and H9N2. Codon-optimization of $\mathrm{HA} 2$ as well as the use of $\mathrm{CD} 40 \mathrm{~L}$ as a targeting ligand/molecular adjuvant were indispensable to enhance HA2-specific mucosal IgA and serum IgG levels. Moreover, induction of HA2-specific T-cell responses was dependent on CD40L, as rAd secreting HA2 subunit without CD40L failed to induce any significant levels of T-cell cytokines. Finally, sera obtained from immunized mice were capable of inhibiting 13 subtypes of influenza A viruses in vitro. These results provide proof of concept for a prototype HA2-based universal influenza vaccine.

\section{INTRODUCTION}

Current seasonal influenza vaccines provide substantial protection by inducing strain-specific neutralizing antibodies (Abs) against the viral hemagglutinin (HA). However, the constantly changing nature of the HA antigenic epitopes presents a daunting challenge to vaccine developers and manufacturers, making it necessary to update the influenza vaccines annually to ensure strains match and thus to achieve sufficient protection in the human population. ${ }^{1-2}$ Although direct human-to-human transmission of avian influenza viruses has been limited so far, the ability of viruses such as $\mathrm{H} 5 \mathrm{~N} 1, \mathrm{H} 7 \mathrm{~N} 7, \mathrm{H} 7 \mathrm{~N} 9$, and $\mathrm{H} 9 \mathrm{~N} 2$ to infect humans and cause disease has raised the concern about their potential to cause devastating pandemics. ${ }^{3-6}$ All of this underscores the importance of developing a universal influenza vaccine capable of inducing broadly protective immunity against the conserved regions of the viral proteins.

The HA protein represents an attractive target for preventive and therapeutic intervention against influenza because it plays critical roles in the early stages of virus infection by binding to viral receptors on target cells and mediating viral and cellular membranes fusion. ${ }^{7} \mathrm{HA}$ is a type I transmembrane homotrimer protein, in which each monomer is composed of two subunits, HA1 and HA2, linked by a single disulfide bond. ${ }^{7}$ The HA1 subunit forms a membrane-distal globular head that contains the receptor-binding site and most of the highly variable immunodominant antigenic regions recognized by neutralizing Abs. ${ }^{8,9}$

\footnotetext{
${ }^{1}$ National Institutes for Food and Drug Control, Beijing, China. ${ }^{2}$ Department of Biopharmaceutics, School of Pharmacy, Fourth Military Medical University, Xi'an, China. ${ }^{3}$ Centre for Vaccine Evaluation, Biologics and Genetic Therapies Directorate, HPFB, Health Canada, Ottawa, Ontario, Canada. ${ }^{4}$ Department of Medical Microbiology and Parasitology, Faculty of Medicine, King Abdulaziz University, Jeddah, Saudi Arabia. ${ }^{5}$ Special Infectious Agents Unit, King Fahd Medical Research Center, King Abdulaziz University, Jeddah, Saudi Arabia. ${ }^{6}$ Department of Biochemistry, Microbiology and Immunology, University of Ottawa, Ottawa, Ontario, Canada. ${ }^{7}$ Shanghai Institute of Biological Products, Shanghai, China and ${ }^{8}$ National Microbiology Laboratory, Public Health Agency of Canada, Winnipeg, Manitoba, Canada. Correspondence: X Li or J Wang (sean.li@hc-sc.gc.ca or wangjz@nicpbp.org.cn)

${ }^{9}$ The first two authors contributed equally to the work.
} 
On the other hand, HA2 forms most of the highly conserved stem-like structure, which anchors the globular domain to the viral membrane and contains the viral fusion peptide. ${ }^{10,11}$

Several cross-subtype broadly neutralizing Abs directed against the stem-like domain of the viral HA have been isolated from human antibody libraries, and the structural basis of their binding and neutralization capabilities has been elucidated. ${ }^{12-17}$ However, induction of such Abs by vaccination has been associated with limited success. For example, while different novel constructs based on the structural data obtained from these broadly neutralizing Abs studies were investigated; they mainly conferred subtype-specific immunity with limited heterosubtypic protection. ${ }^{18-20}$ Thus, it would be of great interest to consider molecular adjuvants such as cytokines and costimulatory molecules to enhance immunogenicity and potency of the relatively weak HA2 antigen.

One of the key molecules that regulate humoral and cellular immune responses is CD40 and its ligand (CD40L). ${ }^{21-23} \mathrm{CD} 40$ is constitutively expressed on all antigen presenting cells, and CD40L is a type II transmembrane multimer protein mainly expressed by activated $\mathrm{CD} 4^{+} \mathrm{T}$ cells transiently as cell-surface or secreted protein. ${ }^{21-23}$ The critical roles of CD40-CD40L interaction in activation of antigen presenting cells, $\mathrm{T}$ cells, and $\mathrm{B}$ cells as well as immune response induction have been demonstrated in many studies. ${ }^{24-28}$

The potential of CD40L as a molecular adjuvant has been also investigated by several groups using different strategies and proven to enhance the immune responses and protection against various pathogens and tumors. ${ }^{29-39}$ Although few reports have employed CD40L to enhance immune response against influenza virus using the full-length HA protein or the highly variable domain of the protein in avian hosts, ${ }^{40,41}$ these studies have mainly focused on immune response induction and only showed partial subtype-specific protection without any evidence of universal protection. Furthermore, although the HA2 subunit is highly conserved among the different subtypes of influenza A viruses and may serve as a "universal" influenza vaccine candidate, it appears to be of low immunogenicity and protective efficacy as Abs induced following immunization with HA protein are found to be mainly targeting the HAl subunit of the proteins. ${ }^{12-20}$ In this study, we investigated the potential and breadth of CD40L as a targeting ligand and molecular adjuvant in enhancing HA2induced protective immune response. Here, we report that intranasal delivery of the vectored vaccine expressing the fusion protein of HA2 and CD40L completely protect mice against lethal challenges with multiple mouse-adapted viruses and sera obtained from the immunized mice could substantially inhibit 13 subtypes of influenza A virus.

\section{RESULTS}

\section{Construction of rAd vaccines and protein expression}

We generated recombinant adenovirus ( $\mathrm{rAd}$ ) vector expressing the wild-type (wt) or codon-optimized (Opt) HA2 from influenza A/California/7/2009(H1N1) virus fused to a secretion signal (S) at the $\mathrm{N}$ terminus and to a trimerized form of murine CD40L ectodomain at the C terminus (rAd-SHA2(wt)FCD40L and rAd-SHA2(Opt)FCD40L, respectively); thus influenza HA2 can be secreted from infected cells and targeted to CD40 receptors on antigen presenting cells (Figure 1a). In addition, we generated rAd vectors expressing non-targeted but secreted monomeric wt HA2 (rAd-SHA2(wt)) or Opt HA2 (rAdSHA2(Opt)). A rAd encoding a non-targeted but secreted trimeric Opt HA2 was also generated (rAd-SHA2(Opt)F) as well as an "empty" vector control (rAd-Control). Prior to animal experiments, the expression of all fusion proteins SHA2(Opt)FCD40L, SHA2(wt)FCD40L, SHA2(Opt)F, SHA2(Opt), and SHA2(wt) was confirmed in vitro (Figure 1b). Western blot analysis also confirmed that only gene products containing the trimerization motif from rAd-SHA2(Opt)FCD40L, rAd-SHA2(wt)FCD40L,

\section{a}

\begin{tabular}{|c|c|c|c|c|c|c|}
\hline rAd-SHA2(Opt)FCD4OL & CMV & $S$ & HA2(Opt) & $\mathrm{F}$ & CD40L & Poly A \\
\hline rAd-SHA2(wt)FCD4OL & CMV & $\mathrm{s}$ & HA2 (wt) & $\mathrm{F}$ & CD40L & Poly A \\
\hline$r A d-S H A 2(O p t) F$ & CMV & $S$ & HA2(Opt) & $\mathrm{F}$ & Poly A & \\
\hline rAd-SHA2(Opt) & CMV & S & HA2(Opt) & & Poly A & \\
\hline rAd-SHA2(wt) & CMV & $\mathrm{s}$ & HA2 (wt) & & Poly A & \\
\hline rAd-Control & CMV & & ly A & & & \\
\hline
\end{tabular}

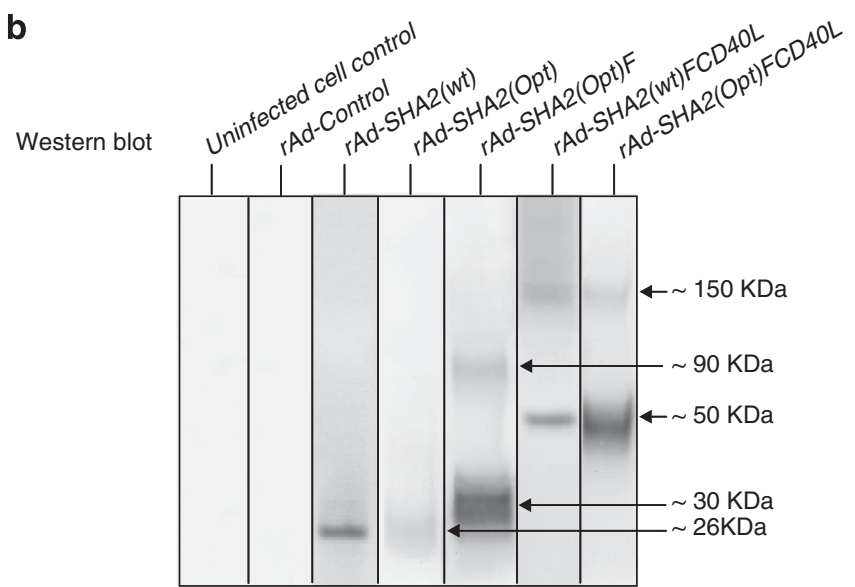

Figure 1 Recombinant Ad constructs and in vitro protein expression. (a) Schematic representation of the generated rAd constructs. The rAd-SHA2(Opt)FCD40L and rAd-SHA2(wt)FCD40L were generated to express secreted HA2(Opt)-FCD40L and HA2(wt)-FCD40L fusion proteins, respectively. $\mathrm{S}$ is an $\mathrm{N}$-terminal leader sequence derived from human tyrosinase signal peptide. HA2 is the HA2 subunit from influenza $\mathrm{A} /$ California/7/2009 virus(H1N1). $\mathrm{F}$ is a 27 amino-acid fragment from the T4 bacteriophage fibritin trimerization motif fused with ectodomain (144 amino acids) of the mouse CD40L. The rAd-SHA2(Opt)F expresses secreted trimeric HA2 protein. The rAd-SHA2(wt) and rAd-SHA2(Opt) express secreted monomeric $\mathrm{HA} 2$ (wt) and HA2(Opt), respectively. The rAd-control is an "empty" control vector. Constructs were engineered in recombinant $\Delta \mathrm{E} 1 / \mathrm{E} 3$ adenovirus $\mathrm{pAdxsi}$ vectors under the control of $\mathrm{CMV}$ promoter. (b) In vitro protein expression in cell culture. Confluent HeLa cells were infected with rAds at multiplicity of infection of 100 and $48 \mathrm{~h}$ later, cell lysates were collected. Protein expression was confirmed by western blot using anti-HA2 polyclonal antibodies. 
and rAd-SHA2(Opt)F show bands at low and high molecular weights corresponding to the expected molecular sizes of the monomeric and trimeric proteins. Specifically, the proteins without the trimerization motif encoded by rAd-SHA2(wt) or rAd-SHA2(Opt) were found to be at a size around $26-28 \mathrm{KDa}$ corresponding to monomeric protein only. On the other hand, the protein encoded by $\mathrm{rAd}-\mathrm{SHA}(\mathrm{Opt}) \mathrm{F}$ was found to form a low molecular weight protein with a band size of $28-30 \mathrm{KDa}$ as well as a high order molecular weight protein with a size around $85-90 \mathrm{KDa}$ corresponding to monomeric and trimeric proteins, respectively. Similarly, western blot analysis of the proteins expressed by rAd-SHA2(wt)FCD40L and rAdSHA2(Opt)FCD40L revealed a low molecular weight band corresponding to the monomeric form of the protein and a high molecular weight trimeric protein with size around $150 \mathrm{KDa}$. In addition, only proteins containing CD40L were detected by anti-CD40L Abs but all proteins were detected by anti-HA2 Abs (data not shown).

\section{CD40L and codon-optimization enhance the immunogenicity of HA2 protein}

In order to evaluate the effects of using CD40L as an adjuvant and targeting molecule on the induction of HA2-specific immune response, mice were intranasally immunized in a prime-boost regimen with different doses $\left(10^{9}, 10^{8}\right.$, or $\left.10^{7} \mathrm{pfu}\right)$ of the generated constructs and the immune response was analyzed 2 weeks post primary and secondary vaccination. Although mice immunized with any of the three constructs expressing HA2 generated significant levels of local and systemic anti-HA2 Abs compared with control mice immunized with rAd-Control or phosphate-buffered saline (PBS) in a doseresponse manner, the codon-optimized construct resulted in the highest titers of circulatory Abs and mucosal IgA (Figure 2 and
Supplementary Figures S1 and S2 online). Specifically, targeting the secreted $\mathrm{HA} 2$ (wt) via CD40L elicited $\geq 2$ fold increase in the levels of mucosal (IgA) and systemic (IgG1 and IgG2a) antibody titer compared with the untargeted rAdSHA2 (wt) control after boosting (Figure 2). In mice immunized with rAd-SHA2(Opt)FCD40L, highly significant levels of nasal IgA and circulatory IgG1 and IgG2a Abs with titers greater than fourfold compared with rAd-SHA2(wt) were also generated. It is also of note that the level of antigen-specific Abs elicited by rAd-SHA2(Opt)FCD40L were significantly more than those induced by rAd-SHA2(wt)FCD40L by $\geq 2$ folds (Figure 2). Similarly, highly significant levels of mucosal IgA and circulatory IgG1 and IgG2a were also observed 2 weeks post secondary immunization with a dose of $10^{8} \mathrm{pfu}$ but not $10^{7} \mathrm{pfu}$ of these constructs (Supplementary Figure S1). Furthermore, the levels of anti-HA2 IgG1 and IgG2a Abs were still highly elevated up to 2 months post secondary immunization (Supplementary Figure S2). These results indicate that both CD40-targeting and HA2 codon-optimization significantly enhanced nasal and circulatory Ab responses.

Antigen-specific T-cell responses were also evaluated by ELISpot to determine the effects of CD40L on cellular immune response 2 weeks post primary and secondary immunization. To our surprise, immunization of mice with rAd-SHA2(wt) did not induce any significant levels of IFN- $\gamma$, TNF- $\alpha$, or IL-4 compared with control groups (rAd-Control or PBS) after priming or boosting (Figure 3 and Supplementary Figure S3). On the other hand, targeting secreted HA2(wt) or HA2(Opt) via CD40L elicited highly significant levels of these cytokines at both time points in a dose-response fashion (Figure 3 and Supplementary Figure S3). Interestingly, there was no significant difference between these two groups suggesting that CD40L preferentially enhanced the induction of cellular a

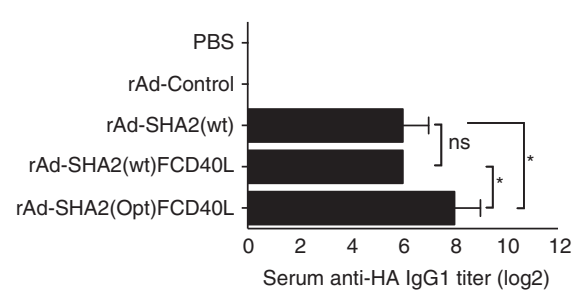

b



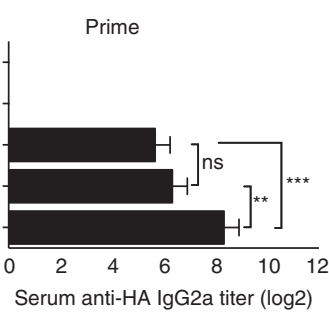

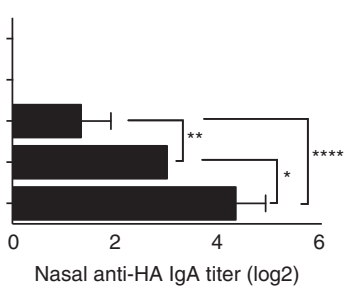

Boost

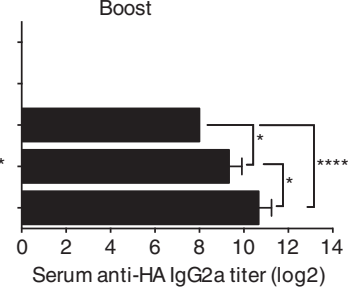

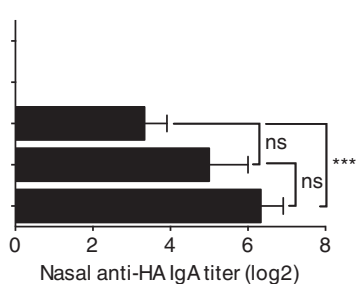

Figure 2 CD4OL and HA2 codon-optimization enhance circulating and nasal anti-HA2 antibody response. HA2-specific antibody titers at 2 weeks after (a) priming and (b) boosting are shown for circulating IgG1 and IgG2a, and nasal IgA. Balb/c mice were intranasally immunized with $10^{9}$ pfu of each rAd virus or with PBS on day 0 and boosted 2 weeks post primary immunization. Data are shown as mean titer \pm s.d. from one experiment out of three independent experiments, with $n=3$ mice per treatment group in each experiment. ${ }^{* * *} P<0.0001$, ${ }^{* * *} P<0.001,{ }^{* *} P<0.01$ and ${ }^{*} P<0.05$ (one-way ANOVA with Bonferroni post-test). 
a
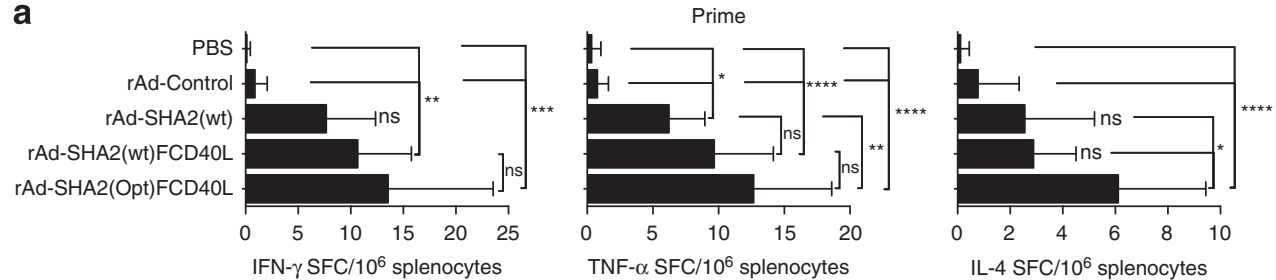

b
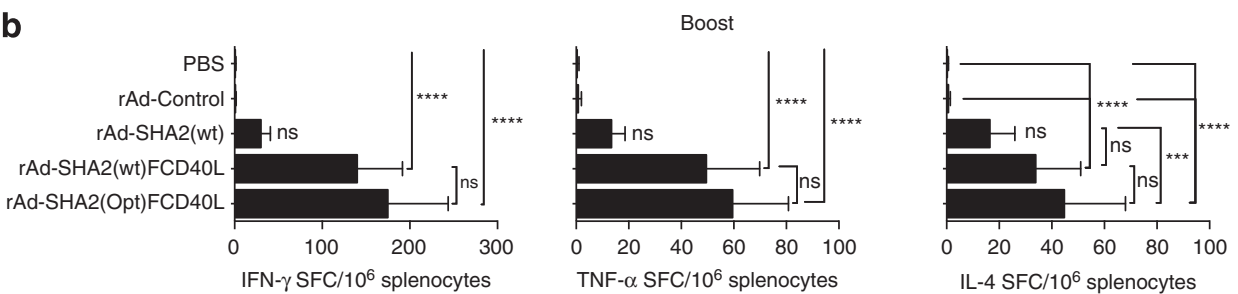

Figure $3 \mathrm{CD} 40 \mathrm{~L}$ enhances T-cell responses to HA2. Balb/c mice were intranasally immunized twice with $10^{9}$ pfu of each rAd virus or with PBS. Mice were sacrificed at 2 weeks (a) post primary or (b) secondary immunization and splenocytes were isolated for IFN- $\gamma$, TNF- $\alpha$, and IL-4 measurement by ELISpot. Data shown as mean numbers of spot-forming cells (SFCs) \pm s.d. from one experiment with $n=3$ per treatment group. Each sample was tested in triplicates. ${ }^{\star * \star \star} P<0.0001,{ }^{* \star \star} P<0.001$, ${ }^{\star \star} P<0.01$ and ${ }^{\star} P<0.05$ (one-way ANOVA with Bonferroni post-test).

immune responses in vaccinated mice. Here, targeting HA2 protein via CD40L always induced higher levels of IFN- $\gamma$ followed by TNF- $\alpha$ and finally IL-4. Collectively, these results indicate that the use of $\mathrm{CD} 40 \mathrm{~L}$ as targeting molecule in conjunction with codon-optimization of the antigen could substantially enhance humoral and cellular immune responses.

\section{Targeting secreted HA2 via CD40L confers heterosubtypic protection in vivo}

Having observed the enhanced immune responses in mice immunized with CD40-targeted HA2, we investigated the potential of these vaccines to provide cross-protection against influenza viruses from different HA subtypes. Immunized mice were challenged 2 weeks following boosting by intranasal administration with $5 \times \mathrm{LD}_{50}$ of mouse-adapted influenza A/Puerto Rico/8/1934(H1N1), A/Jingke/30/95(H3N2) or the avian $\mathrm{A} /$ chicken/Jiangsu/7/2002(H9N2) strains. As shown in Figure 4, although immunization of mice with rAd-SHA2(wt) at a dose of $10^{9} \mathrm{pfu}$ provided $50-60 \%$ protection against the same HA group 1 viruses, i.e., A/Puerto Rico/8/1934(H1N1), and $\mathrm{A} /$ chicken/Jiangsu/7/2002(H9N2) strains, only $30 \%$ protection was observed against the $\mathrm{H} 3 \mathrm{~N} 2$ virus, which belongs to HA group 2 (Figure 4). Importantly, the same dose $\left(10^{9} \mathrm{pfu}\right)$ of either rAd-SHA2(wt)FCD40L or rAd-SHA2(Opt)FCD40L conferred complete protection against all the three viruses regardless of the subtype (Figure 4).

Such protective effects were observed in a dose-response fashion as lower amounts of antigens correlated with decreasing levels of protection, with the CD40-targeted vaccines consistently affording better protection than the untargeted HA. Specifically, one $\log$ less of the two targeted vaccines still provided complete protection against both $\mathrm{H} 1 \mathrm{~N} 1$ and the avian H9N2 viruses (Supplementary Figure S4). However, rAdSHA2(Opt)FCD40L afforded the best protection against the more phylogenetically distant $\mathrm{H} 3 \mathrm{~N} 2$ virus (60\% protection) while rAd-SHA2(wt)FCD40L protected $30 \%$ of the mice only (Supplementary Figure S4). The strong protection by rAdSHA2(Opt)FCD40L was further confirmed by the superior protection against all three viruses compared with rAdSHA2(wt)FCD40L and rAd-SHA2(wt) when a very low dose of $10^{7} \mathrm{pfu}$ was employed for immunization ( Supplementary Figures S5). Observation of weight loss also revealed that mice vaccinated with rAd-SHA2(Opt)FCD40L always maintained more weight after challenge than those immunized with other constructs (Figure 4 and Supplementary Figures S4 and S5).

We next determined the level of virus titers in the lung and nasal tissues. As shown in Figure 5, immunization with rAdSHA2(wt)FCD40L and rAd-SHA2(Opt)FCD40L showed $\geq 3$ $\log , 2 \log$, and $1.5 \log$ reduction in H1N1, H9N2, and H3N2 lung and nasal viral loads compared with control groups, respectively. On the other hand, viral titers in mice immunized with rAd-SHA2(wt) were reduced by $\leq 1.5 \log$ as compared with control groups. Taken together, these data demonstrate that not only CD40L but also HA2 codon-optimization substantially enhanced the protective efficacy of HA2 and significantly promoted heterosubtypic immunity against $\mathrm{H} 1 \mathrm{~N} 1$ strain as well as the divergent viruses, which belong to the phylogenetically distinct $\mathrm{H} 3$ and avian $\mathrm{H} 9$ subtypes.

\section{Targeting codon-optimized HA2 via CD40L elicits broadly inhibitory Abs against 13 subtypes of influenza $A$ virus}

As all of the vaccine constructs were made using HA2 subunit of H1N1, we next wished to determine whether sera obtained from mice immunized with the various vaccine constructs could inhibit 13 different subtypes of viruses available in our laboratories (H1-13). It needs to be mentioned that the other three subtypes (H14-16) are very rare and also not available to us. ${ }^{11}$ Here, we used an in vitro microneutralization assay to determine whether the sera from immunized mice could inhibit all the subtypes and whether there is any difference between the 
a
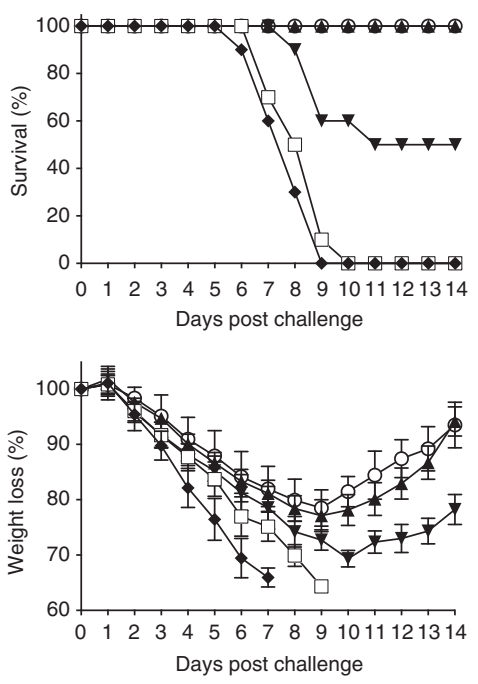

b
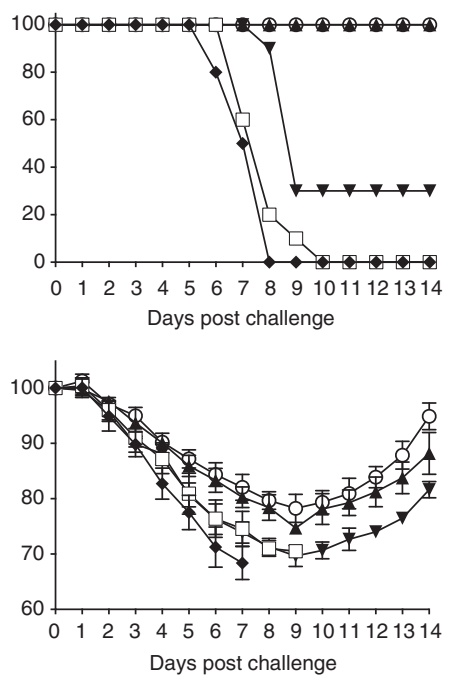

C $\mathrm{A} /$ chicken/Jiangsu/7/2002(H9N2)


Figure 4 Targeting wild-type or codon-optimized HA2 via CD40L provides complete heterosubtypic protection. Survival curves and body weight loss of $\mathrm{Balb} / \mathrm{c}$ mice immunized with two doses of $10^{9} \mathrm{pfu}$ of the indicated rAd constructs and challenged with $5 \times \mathrm{LD}_{50}$ of mouse-adapted influenza (a) A/Puerto Rico/8/1934(H1N1) (b) A/Jingke/30/95(H3N2) or (c) A/chicken/Jiangsu/7/2002(H9N2) viruses 2 weeks post secondary immunization. Data are shown from one experiment with $n=10$ mice per treatment group.

a Lung viral titer

A/Puerto Rico/8/1934(H1N1)

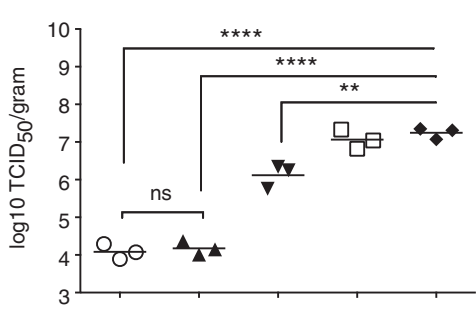

b Nasal viral titer

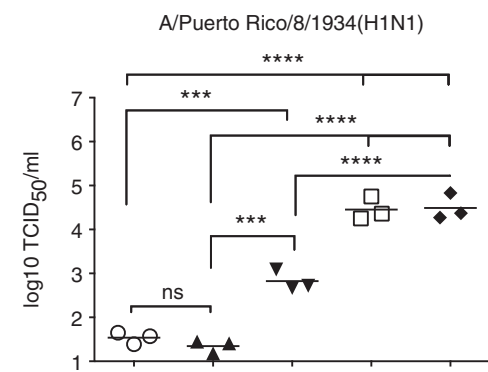

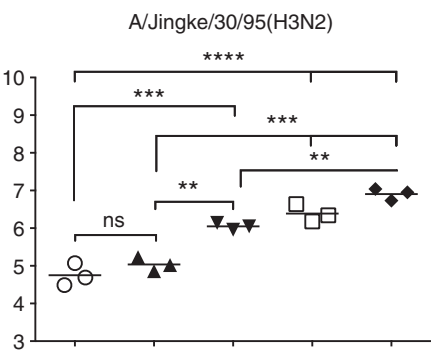

A/Jingke/30/95(H3N2)

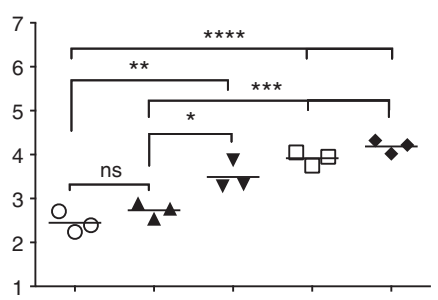

A/chicken/Jiangsu/7/2002(H9N2)

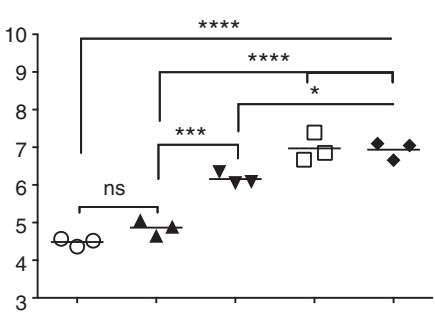

rAd-SHA2(Opt)FCD40L

$\Delta$ rAd-SHA2(wt)FCD40L

$\nabla$ rAd-SHA2(wt)

$\square$ rAd-Control

PBS

Figure 5 CD40-targeted HA2 reduces lung and nasal viral titers in immunized mice. (a) Lung and (b) nasal titers were evaluated on day 5 post challenge from Balb/c mice immunized with two doses of $10^{9}$ pfu of the indicated $r A d$ constructs and challenged with $5 \times L_{50}$ of mouse-adapted influenza $A / P u e r t o$ Rico/8/1934(H1N1), A/Jingke/30/95(H3N2) or A/chicken/Jiangsu/7/2002(H9N2). Data are shown from one experiment with $n=3$ per treatment group. ${ }^{\star \star \star \star} P<0.0001,{ }^{\star \star \star} P<0.001,{ }^{\star \star} P<0.01$ and ${ }^{\star} P<0.05$ (one-way ANOVA with Bonferroni post-test).

sera obtained from mice immunized with the targeted or untargeted vaccines. As shown in Figure 6, only sera collected from mice immunized with rAd-SHA2(Opt)FCD40L showed potent cross-inhibitory activity against 13 HA subtypes, whereas sera collected from rAd-SHA2(wt)FCD40L-vaccinated mice demonstrated protective activity against most subtypes except for $\mathrm{H} 4$ and $\mathrm{H} 5$ strains. Surprisingly, antisera collected from rAd-SHA2(wt) immunized mice failed to inhibit any of the tested subtypes. These results clearly underscore the role of CD40L and codon-optimization in enhancing the HA2-based immune response and the induction of broadly inhibitory Abs against all influenza A viruses. 

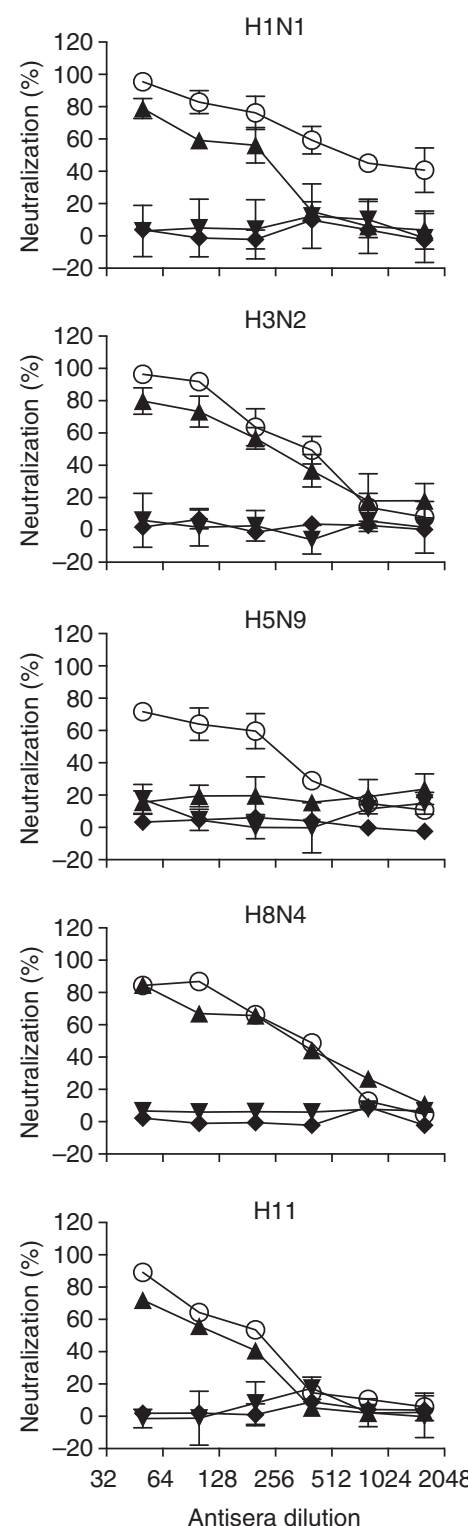
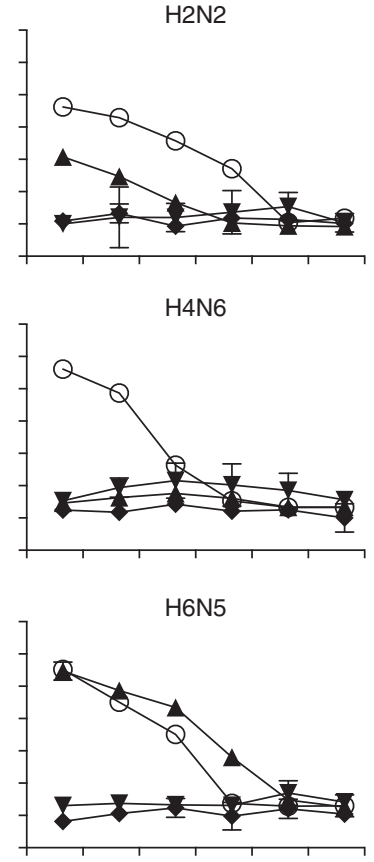

H9N2

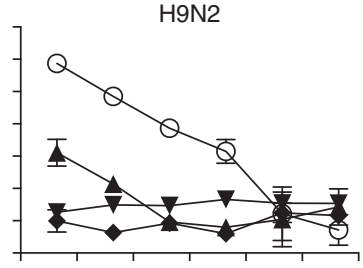

H12N6

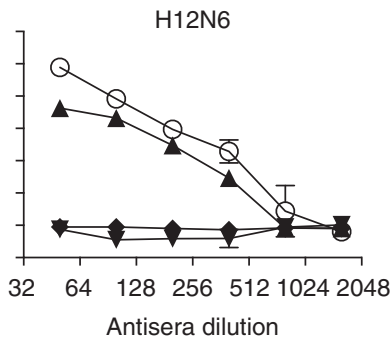

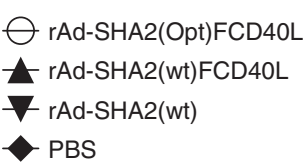

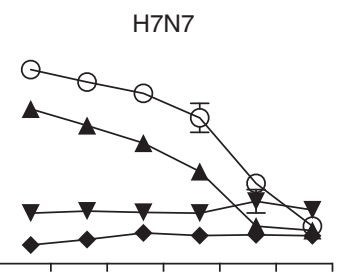

H10N8

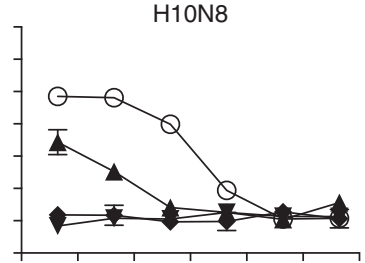

H13

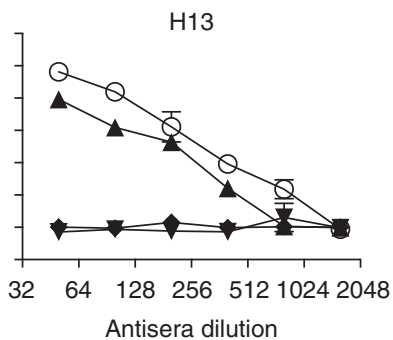

Figure 6 Antisera from mice immunized with rAd-SHA2(Opt)FCD40L cross-neutralize diverse influenza strains from group 1 and group 2 viruses. Pooled sera from three mice immunized with $10^{9} \mathrm{pfu}$ of each $\mathrm{rAd}$ virus were tested against several influenza $\mathrm{A}$ viruses. Data are presented as percentage of neutralization \pm s.d. from triplicates.

\section{CD40L but not the trimerization motif enhances the breadth of HA2-based protection}

Having observed the robust protective effects of rAdSHA2(Opt)FCD40L against diverse subtypes, we next wished to determine whether the trimerization motif rather than the $\mathrm{CD} 40 \mathrm{~L}$ is responsible for the enhanced immune response we observed. To this end, rAd-SHA2(Opt) and rAd-SHA2(Opt)F were compared with rAd-SHA2(Opt)FCD40L. As shown in Figure $7 \mathbf{a}-\mathbf{c}$, no significant differences in animal survival rate or weight loss were observed between rAd-SHA2(Opt) and rAdSHA2(Opt)F, suggesting that the trimerization motif itself cannot enhance the protection regardless of the trimeric fusion protein encoded by rAd-SHA2(Opt)F. In contrast, although it did not prevent infection and weight loss, the addition of the
CD40L using rAd-SHA2(Opt)FCD40L resulted in 100\% protection of the animals against the three influenza subtypes (Figure $7 \mathbf{a}-\mathbf{c})$. Moreover, these protection effects correlated well with the antibody and cytokine levels in these immunized mice (Figure $\mathbf{7 d - e}$ ). Similar results were also observed when mice were immunized with lower doses of each $\mathrm{rAd}$ (Supplementary Figure S6). Taken together, these data provide strong supporting evidence on the effects of CD40L as an adjuvant in inducing enhanced immunological protection against diverse strains of influenza A virus.

\section{DISCUSSION}

Current influenza vaccines provide substantial protection in humans, however they mainly induce strain-specific 
a
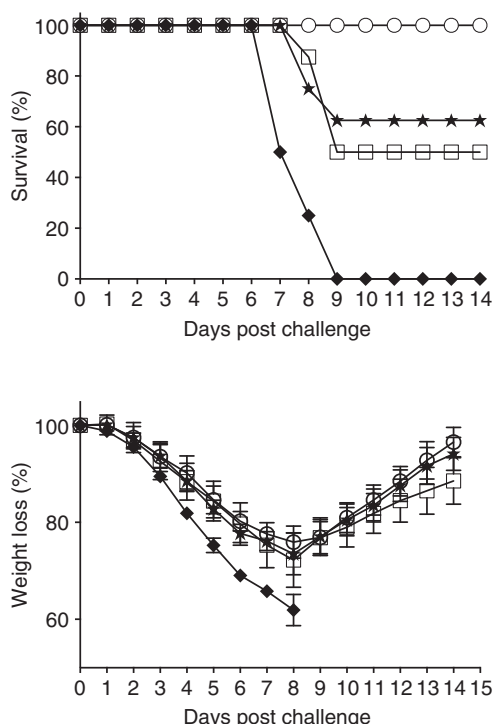

b
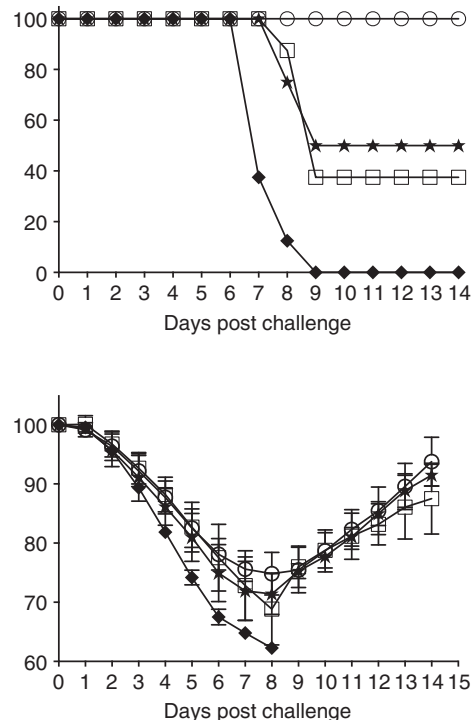

C A/chicken/Jiangsu/7/2002(H9N2)

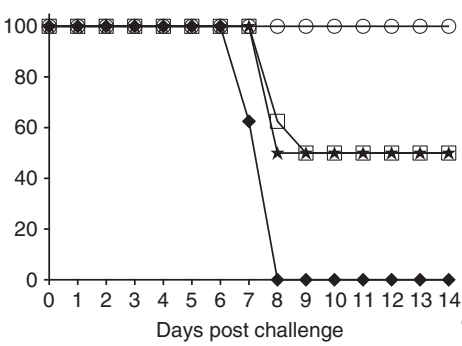

rAd-SHA2(Opt)FCD40I

$\star$ rAd-SHA2(Opt)F

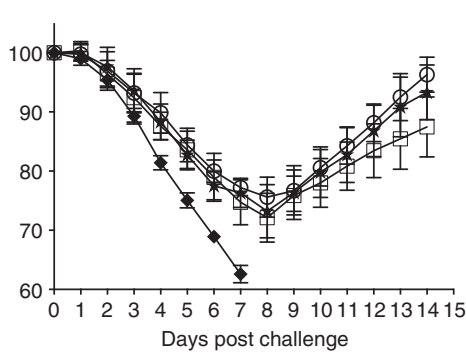
$\square$ rAd-SHA2(Opt)

d
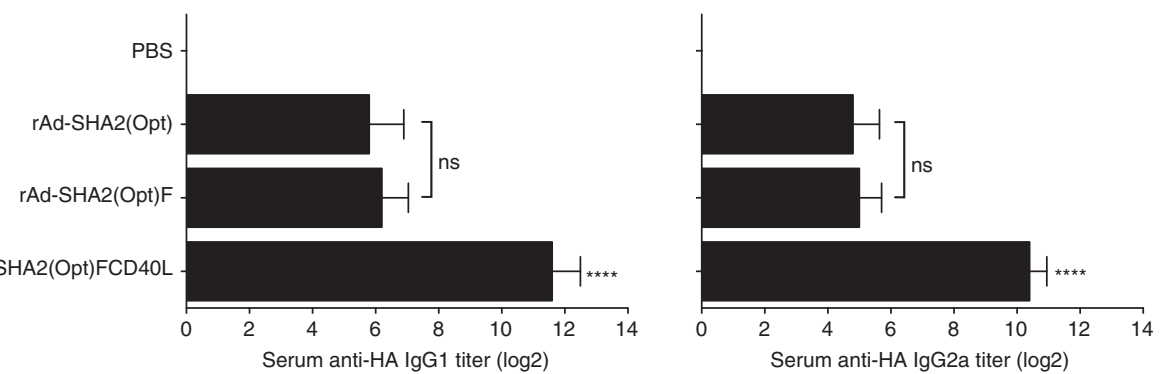

e
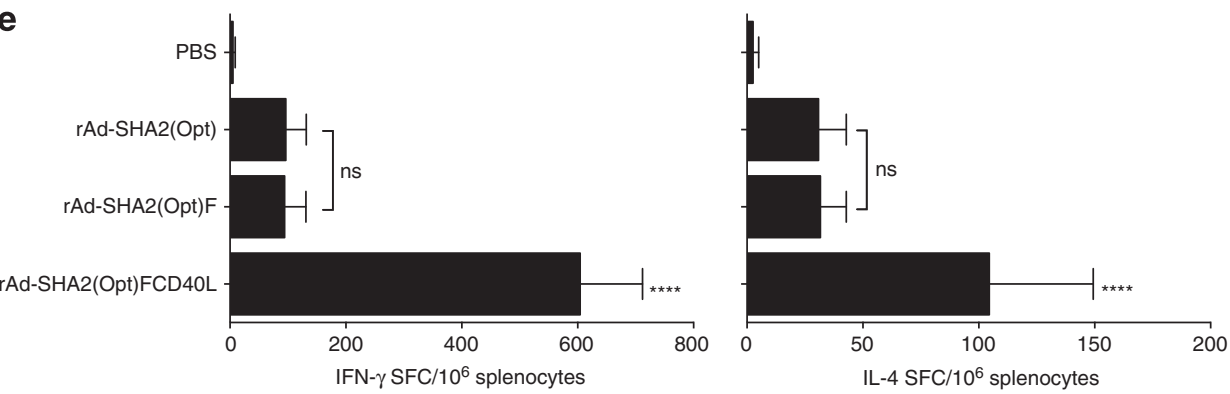

Figure 7 Enhanced protection and immune response is dependent on CD40L. Survival curves and body weight loss of Balb/c mice immunized with two doses of $10^{9} \mathrm{pfu}$ of the indicated rAd constructs and challenged with $5 \times \mathrm{LD}_{50}$ of mouse-adapted influenza (a) A/Puerto Rico/8/1934(H1N1) (b) A/Jingke/ 30/95(H3N2) or (c) A/chicken/Jiangsu/7/2002(H9N2) viruses 2 weeks post secondary immunization. Circulating HA2-specific IgG1 and IgG2a titers (d) and IFN- $\gamma$ and IL-4T cell responses (e) are shown at 2 weeks post secondary immunization. Data are shown from one experiment with $n=8$ mice per treatment group in (a-c). Data in (d) are shown as mean titer \pm s.d. from one experiment, with $n=5$ mice per treatment group. Data in (e) are shown as mean numbers of spot-forming cells (SFCs) \pm s.d. from one experiment with $n=5$. Each sample was tested in triplicates. ${ }^{* * * *} P<0.0001$ (one-way ANOVA with Bonferroni post-test).

neutralizing Abs. ${ }^{1,42}$ Most of these Abs target the highly variable regions of the globular head in the HA1 subunit; therefore, they cannot induce heterosubtypic immunity. ${ }^{1,15}$ The globular head in the HA1 subunit of the virus is highly immunogenic and therefore elicits high levels of neutralizing Abs, which block viral attachment to host cell receptors. On the other hand, the HA2 subunit that represents most of the HA stem region show high sequence conservation among the different HA subtypes. Therefore, the HA2 region would be a very attractive target to induce Abs of broad neutralizing activity. $^{12-20}$ However, previous studies suggest that the immungenecity of the HA2 is very weak, ${ }^{43}$ and the low levels of the Abs in patients may not be sufficient to provide protection. $^{43-45}$

In this communication, we described a novel "universal" influenza vaccine based on the HA2 subunit, in which we utilized CD40L, a key linker between the innate and the adaptive immune responses, not only as an adjuvant but also as 
a targeting molecule to efficiently stimulate antigen presenting cells and to enhance HA2-specific immune responses. The data presented here clearly demonstrate that using CD40L augmented $\mathrm{HA} 2$-specific immune responses and enhanced its protective efficacy. Specifically, although codon-optimization of HA2 seems to induce significantly higher levels of local and systemic anti-HA2 Abs, targeting secreted wild-type or codon-optimized HA2 via CD40L significantly promoted Abs secretion and HA2specific cytokines production by $\mathrm{T}$ cells compared with untargeted HA2. These results also indicate that the secreted fusion protein HA2-FCD40L apparently retain the capability to elicit cell-mediated immune responses as demonstrated by the enhanced HA2-specific cytokine production by T cells in mice immunized with constructs containing CD40L only.

It is noteworthy that although the HA2 gene of influenza A/California/7/2009(H1N1) virus was used to generate our constructs, effective protection was still achieved in animals challenged with $\mathrm{H} 3$ virus, which shares $\sim 50 \%$ of sequence homology with A/California/7/2009(H1N1) virus. Moreover, the better protection against A/Puerto Rico/8/1934(H1N1) and avian $\mathrm{A} /$ chicken/Jiangsu/7/2002(H9N2), particularly at lower doses of the constructs, is in agreement with the fact that the HA2 subunits of these two viruses share higher homology with that of A/California/7/2009(H1N1), i.e., $92 \%$ and 65\% identity, respectively.

The universality of this prototype vaccine is also supported by results generated from our in vitro experiments in which 13 different subtypes of influenza A virus (H1-13) were tested. Most of the 13 viruses are not mouse-adapted, therefore cannot be used in animal challenge studies. However, antisera from the CD40-targeted immunogens, particularly the rAdSHA(Opt)FCD40L construct but not rAd-SHA(wt), were able to significantly inhibit all 13 different subtypes. These data collectively suggest that the head domain of HA (mainly HA1 subunit) masks the recognition of the highly conserved regions in the stem of the HA protein (HA2) by the immune system in addition to the low immunogenicity of $\mathrm{HA} 2,{ }^{43-45}$ but CD40L as targeting molecule and molecular adjuvant could overcome the low immunogenicity of the HA2 subunit and can lead to universal protection against divergent influenza strains belonging to group 1 and $2 \mathrm{HA}$ subtypes.

Of note, antisera collected from rAd-SHA2-immunized mice failed to show any inhibitory effect in vitro regardless to the observed partial in vivo protection and reduced lung virus titers. This could be due to the monomeric form or the improper folding of SHA2 protein expressed by this construct in the absence of the T4 fibritin especially that some BnAb such as the F16 Ab mediates their inhibitory effect via binding to conformational epitopes in the HA stem trimer. ${ }^{16}$ However, the data shown in Figure 7 and Supplementary Figure S6 clearly show that immunization with rAd-SHA2(Opt)F cannot elicit any significant enhancement in protection or immune response induction compared with rAd-SHA2(Opt) although it can be expressed as a trimer. This clearly suggests that using $\mathrm{CD} 40 \mathrm{~L}$ is the main factor that is enhancing the protection, immunogenicity, and induction of broadly inhibitory Abs.
Furthermore, the in vitro data shown in Figure 6 suggest the induction of broadly inhibitory Abs by CD40-targted HA2. However, the animal studies clearly demonstrate that immunization of mice by HA2 targeted via CD40L elicited humoral and cellular immune responses and prevented death but not infection (immunized mice lost nearly $20 \%$ of their weight upon challenge). This is consistent with the fact that most antiHA2 stem region Abs do not prevent viral entry but interfere with downstream steps in viral replication ${ }^{12-17}$ in contrast to strain-specific neutralizing Abs that target the head domain of HA and completely prevent infection at the viral attachment step. In addition, the in vivo protection observed in this study is similar to the non-sterilizing immunity conferred by internal viral proteins such as the nucleoprotein in which cellular immunity as well as non-neutralizing anti-nucleoprotein Abs have been implicated in protection. ${ }^{42,46}$ Experiments involving passive transfer of antisera or adoptive transfer of $\mathrm{T}$ cells from vaccinated mice into naive animals could provide more mechanistic details and elucidate the role and the importance of both arms of the immune system in protection. However, the induction of enhanced antigen-specific $\mathrm{T}$-cell cytokines and $\mathrm{Ab}$ responses, the presence of broadly inhibiting Abs and the protection of mice from death upon vaccination with rAd expressing CD40-targeted HA2 compared with other groups do not only indicate that both cellular and humoral immune response are involved but also suggest that protection was dependent on targeting HA2 protein to CD40-expressing cells via CD40L.

In short, the HA2 has the potential of being a candidate "universal" vaccine but is unlikely to induce sufficient protective immune responses. Expression of the HA2 as a secreted form of fusion protein with CD40L could substantially increase the immunogenicity of HA2 and elicit broadly inhibitory Abs against most influenza A subtypes.

\section{METHODS}

Cell lines and viruses. Madin-Darby canine kidney cells, HeLa cells, and HEK-293A cells were cultured in complete Dulbecco's modified Eagle's medium with $10 \%$ heat-inactivated fetal bovine serum as described previously. ${ }^{47}$ The mouse-adapted influenza A/Puerto Rico/ 8/1934(H1N1), A/Jingke/30/95(H3N2), and A/chicken/Jiangsu/7/ 2002(H9N2) viruses were obtained from Shanghai Institute of Biological Products. All viruses were propagated at $37^{\circ} \mathrm{C}$ in the allantoic cavities of 10-day-old embryonated hen eggs for 3 days. Allantoic fluid was clarified by centrifugation, aliquoted, and stored at $-80{ }^{\circ} \mathrm{C}$. Viruses were titrated in Madin-Darby canine kidney cells and used for viral challenge. Median lethal dose $\left(\mathrm{LD}_{50}\right)$ for each virus in $\mathrm{Balb} / \mathrm{c}$ mice were determined using Reed-Muench formula. ${ }^{48}$

Generation of rAds. Constructs were designed to express a secreted form of wild-type, rAd-SHA2(wt), or codon-optimized, rAd-SHA2(Opt), influenza HA2 subunit (amino acids 345-566) from influenza A/California/7/2009(H1N1) virus (GenBank Acc. no. ACP41105) fused to 23 amino acids (MLLAVLYCLLWSFQTSAGHFPRA) from the human tyrosinase signal peptide (GenBank Acc. no. AH003020) at the $\mathrm{N}$ terminus as previously described. ${ }^{49}$ Other constructs, rAdSHA2(wt)FCD40L and rAd-SHA2(Opt)FCD40L, were also generated to express SHA2(wt) and SHA2(Opt) fused to a 27 amino-acid fragment from the bacteriophage T4 fibritin trimerization motif (GYIPEAPRDGQAYVRKDGEWVLLSTFL) connected to the 
ectodomain of mouse CD40L (GeneBank Acc. no. NM_011616) (amino acids 117-260) as previously described. ${ }^{30}$ A rAd expressing secreted HA2(Opt) fused to the T4 fibritin trimerization motif without CD40L (rAd-SHA2(Opt)F) as well as an empty rAd (rAd-Control) were also produced. rAds were generated using pAdxsi Adenoviral Expression System. All fusion genes were joined using overlap PCR and cloned into pShuttle-CMV vector between XhoI/EcoRI sites, which was subsequently co-transformed into Escherichia coli cells $(\mathrm{DHF} \alpha)$ cells with $\triangle \mathrm{E} 1 / \mathrm{E} 3 \mathrm{pAdxsi}$ vector for homologous recombination. The resulting recombinant plasmids were PacI-linreaized and transfected into HEK-293A cells to package the rAd vectors. Recombinant viruses were purified twice by cesium chloride densitygradient centrifugation, titrated, and stored at $-80^{\circ} \mathrm{C}$ until used. Cloning was confirmed by XhoI digestion and PCR. Viruses were titrated by plaque assay in HEK-293A cells.

Protein expression in cell culture. The generated rAds were used to infect confluent HeLa cells in six-well plate at a multiplicity of infection of 100. Forty-eight hours later, protein expression in cell lysates of infected cells was confirmed by western blot of proteins separated on sodium dodecyl sulfate -polyacrylamide gel electrophoresis or native gradient $(4-12 \%)$ polyacrylamide gel electrophoresis as previously described $^{30}$ using anti-CD40L monoclonal Abs and rabbit anti-HA Abs (Sino Biological Inc., Beijing, China).

Animal study. Six to eight-week-old female Balb/c mice were maintained in a pathogen-free animal facility at Shanghai Institute of Biological Products. All animal experiments were conducted in accordance with Institutional Guidelines and with the approval of the Animal Care and Use Committee. Mice were intranasally immunized twice with $10^{7} \mathrm{pfu}, 10^{8} \mathrm{pfu}$, or $10^{9} \mathrm{pfu}$ of each rAd construct (rAdSHA2(Opt)FCD40L, rAd-SHA2(wt)FCD40L, rAd-SHA2(Opt)F, rAd-SHA2(Opt), rAd-SHA2(wt), or rAd-Control) or with PBS. rAds were administrated to mice on day 0 , and mice were boosted with the same dose on day 14 . Serum and nasal washes were collected on days 0 (pre-bleed), 14 and 28 for antibody analysis. Spleens were harvested from some mice on days 14 and 28 and single cell suspensions were prepared for cytokines determination. Two weeks post secondary immunization, Balb/c were intranasally challenged with $5 \times \mathrm{LD}_{50}$ of each influenza virus. After challenge, mice were weighed daily qand clinically monitored for signs of illness for 14 days. Survival data are presented as a percentage of surviving animals at each time point compared with the initial number of animals in each group. Weight loss is expressed as percentage of animal weight at each time point relative to their initial body weight. Lung and nasal washes were collected from three mice in each group 5 days post challenge for viral load determination using median tissue culture infectious doses $\left(\mathrm{TCID}_{50}\right)$.

ELISA. The end-point titers of serum anti-HA2 IgG1 and IgG2a Abs, and IgA in nasal washes collected form immunized mice were determined by ELISA as described previously ${ }^{50}$ with minor modifications. Briefly, 96-well plates were coated with $100 \mu \mathrm{l} /$ well of $8 \mu \mathrm{g} / \mathrm{ml}$ of purified influenza A/California/7/2009(H1N1) virus. Plates were washed with PBS containing $0.05 \%$ Tween 20 (PBS-T) and blocked with $200 \mu \mathrm{l}$ blocking buffer for 1 hour at $37^{\circ} \mathrm{C}$. After washing, $100 \mu \mathrm{l} /$ well of each serum samples from immunized mice at the indicated time points were added in a twofold serial dilution and incubated at $37^{\circ} \mathrm{C}$ for 1 hour. After washing, appropriate horseradish peroxidise-conjugated goat anti-mouse Abs (anti-IgG1, anti-IgG2a, or anti-IgA) were added at 1:1000 dilution for 1 hour at $37^{\circ} \mathrm{C}$. After six washes, $3,3^{\prime}, 5,5^{\prime}-$ tetramethylbenzidine substrate was used for colorimetric development and the absorbance was read at $450 \mathrm{~nm}$. End-point antibody titers were expressed as the reciprocals of the final detectable dilution with a cutoff defined as the mean of pre-bleed samples plus three s.d.

Cytokines ELISpot. ELISpot was performed using Millipore MultiScreen 96-Well Plates (Fisher Scientific, Pittsburgh, PA) and
Mabtech reagents for ELISpot assay of mouse IFN- $\gamma$, TNF- $\alpha$, and IL-4 (Mabtech, Inc., Cincinnati, $\mathrm{OH}$ ) according to manufacturer's protocols. Cells were stimulated with influenza H1N1 virus at $2 \mu \mathrm{g} / \mathrm{ml}$. Spots were developed using Pierce 3,3',5,5'-tetramethylbenzidine substrate (Thermo Fisher Scientific Inc, Rockford, IL) and enumerated with an BIOREADER 6000 counter (BIO-SYS, Kiefernweg, Karben, Germany). Data are presented as spot-forming cells per million cells.

Microneutralization. Microneutralization was carried out as described previously ${ }^{12}$ with slight modifications. Briefly, serum samples obtained from immunized mice were treated with receptor destroying enzyme and used for microneutralization. Approximately 100 TCID $_{50}$ of each viruses (viruses used were previously described ${ }^{11}$ ) were mixed with an equal volume of serially diluted sera pooled from three mice in each group in 96-well plates and incubated for $1 \mathrm{~h}$ at $37^{\circ} \mathrm{C}$. Virus- $\mathrm{Ab}$ mixture was transferred onto confluent monolayers of Madin-Darby canine kidney cells and incubated at $37^{\circ} \mathrm{C}$ for $20 \mathrm{~h}$. Cell monolayers were washed twice with PBS, fixed with ice-cold $80 \%$ acetone for 10 minutes and viral nucleoprotein was detected by indirect ELISA using rabbit anti-nucleoprotein Abs (Sino Biological).

Data analysis. Statistical analysis was conducted using either one-way or two-way analysis of variance when appropriate. Bonferroni posttest was used to adjust for multiple comparisons between the different groups. All statistical analysis was conducted using GraphPad Prism software (San Diego, CA).

SUPPLEMENTARY MATERIAL is linked to the online version of the paper at http://www.nature.com/mi

\section{ACKNOWLEDGMENTS}

A.M.H. is supported by King Abdulaziz University. This work was supported by the Canadian Regulatory Strategy for Biotechnology (X.L.) and Ministry of Science and Technology, P.R. China (2013DFA31680 to J.W. and C.L.).

\section{DISCLOSURE}

The authors declared no conflict of interest.

(c) 2015 Society for Mucosal Immunology

\section{REFERENCES}

1. Gerhard, W., Mozdzanowska, K. \& Zharikova, D. Prospects for universal influenza virus vaccine. Emerging Infect. Dis 12, 569-574 (2006).

2. Hashem, A.M. et al. Recent developments in bioinformatics analyses of influenza A virus surface glycoproteins and their biological relevance. Curr. Bioinform. 6, 415-426 (2011).

3. Subbarao, K. et al. Characterization of an avian influenza A (H5N1) virus isolated from a child with a fatal respiratory illness. Science 279, 393-396 (1998).

4. Lin, Y.P. et al. Avian-to-human transmission of H9N2 subtype influenza A viruses: relationship between $\mathrm{H} 9 \mathrm{~N} 2$ and $\mathrm{H} 5 \mathrm{~N} 1$ human isolates. Proc. Natl. Acad. Sci. USA 97, 9654-9658 (2000).

5. Fouchier, R.A.M. et al. Avian influenza A virus (H7N7) associated with human conjunctivitis and a fatal case of acute respiratory distress syndrome. Proc. Natl. Acad. Sci. USA 101, 1356-1361 (2004).

6. Dai, C. \& Jiang, M. Understanding H7N9 avian flu. B.M.J. 346, f2755 (2013).

7. Skehel, J.J. \& Wiley, D.C. Receptor binding and membrane fusion in virus entry: the influenza hemagglutinin. Annu. Rev. Biochem. 69, 531-569 (2000).

8. Gerhard, W., Yewdell, J., Frankel, M.E. \& Webster, R.G. Antigenic structure of influenza virus haemagglutinin defined by hybridoma antibodies. Nature 290, 713-717 (1981).

9. Wiley, D.C. \& Skehel, J.J. The structure and function of the hemagglutinin membrane glycoprotein of influenza virus. Annu. Rev. Biochem. 56, 365-394 (1987). 
10. Chen, J. et al. A soluble domain of the membrane-anchoring chain of influenza virus hemagglutinin (HA2) folds in Escherichia coli into the low-pHinduced conformation. Proc. Natl. Acad. Sci. USA 92, 12205-12209(1995).

11. Chun, S. et al. Universal antibodies and their applications to the quantitative determination of virtually all subtypes of the influenza A viral hemagglutinins. Vaccine 26, 6068-6076 (2008).

12. Hashem, A.M. et al. Universal antibodies against the highly conserved influenza fusion peptide cross-neutralize several subtypes of influenza A virus. Biochem. Biophy. Res. Commun 403, 247-251 (2010).

13. Throsby, M. et al. Heterosubtypic neutralizing monoclonal antibodies cross-protective against H5N1 and H1N1 recovered from human IgM + memory B cells. PLoS ONE 3, e3942 (2008).

14. Ekiert, D.C. et al. Antibody recognition of a highly conserved influenza virus epitope. Science 324, 246-251 (2009).

15. Sui, J. et al. Structural and functional bases for broad-spectrum neutralization of avian and human influenza A viruses. Nat. Struct. Mol. Biol. 16, 265-273 (2009).

16. Corti, D. et al. A neutralizing antibody selected from plasma cells that binds to group 1 and group 2 influenza A hemagglutinins. Science 333 , 850-856 (2011).

17. Ekiert, D.C. et al. A highly conserved neutralizing epitope on group 2 influenza A viruses. Science 333, 843-850 (2011).

18. Bommakanti, G. et al. Design of an HA2-based Escherichia coli expressed influenza immunogen that protects mice from pathogenic challenge. Proc. Natl. Acad. Sci. USA 107, 13701-13706 (2010).

19. Steel, J. et al. Influenza virus vaccine based on the conserved hemagglutinin stalk domain. mBio 1, e00018-10 (2010).

20. Wang, T.T. et al. Vaccination with a synthetic peptide from the influenza virus hemagglutinin provides protection against distinct viral subtypes. Proc. Natl. Acad. Sci. USA 107, 18979-18984 (2010).

21. van Kooten, C. \& Banchereau, J. CD40-CD40 ligand. J. Leukoc. Biol. 67, 2-17 (2000)

22. Bishop, G.A. \& Hostager, B.S. The CD40-CD154 interaction in B cell-Tcell liaisons. Cytokine Growth Factor Rev. 14, 297-309 (2003).

23. Quezada, S.A., Jarvinen, L.Z., Lind, E.F. \& Noelle, R.J. CD40/CD154 interactions at the interface of tolerance and immunity. Annu. Rev. Immunol. 22, 307-328 (2004).

24. Oxenius, A. et al. CD40-CD40 ligand interactions are critical in T-B cooperation but not for other anti-viral CD4 + Tcell functions. J. Exp. Med. 183, 2209-2218 (1996).

25. Schultze, J.L. et al. CD40-activated human B cells: an alternative source of highly efficient antigen presenting cells to generate autologous antigen-specific $T$ cells for adoptive immunotherapy. J. Clin. Invest. 100, 2757-2765 (1997).

26. Bennett, S.R., Carbone, F.R., Karamalis, F., Flavell, R.A., Miller, J.F. \& Heath, W.R. Help for cytotoxic-T-cell responses is mediated by CD40 signalling. Nature 393, 478-480 (1998).

27. Schoenberger, S.P., Toes, R.E., van der Voort, E.I., Offringa, R. \& Melief, C.J. T-cell help for cytotoxic T lymphocytes is mediated by CD40-CD40L interactions. Nature 393, 480-483 (1998).

28. Bergwelt-Baildon von, M. et al. CD40-stimulated B lymphocytes pulsed with tumor antigens are effective antigen-presenting cells that can generate specific T cells. Cancer Res 2003;63:2836-43. Cancer Res 64, 4055-4056. author reply 4056-7 (2004).

29. Tripp, R.A., Jones, L., Anderson, L.J. \& Brown, M.P. CD40 ligand (CD154) enhances the Th1 and antibody responses to respiratory syncytial virus in the Balb/c mouse. J. Immunol. 164, 5913-5921 (2000).

30. Pereboev, A.V. et al. Enhanced gene transfer to mouse dendritic cells using adenoviral vectors coated with a novel adapter molecule. Mol. Ther. 9 712-720 (2004).

31. Zheng, M. et al. CD4 + T cell-independent DNA vaccination against opportunistic infections. J. Clin. Invest. 115, 3536-3544 (2005)

32. Huang, D. et al. Significant alterations of biodistribution and immune responses in Balb/c mice administered with adenovirus targeted to CD40(+) cells. Gene Ther 15, 298-308 (2008).
33. Liu, J. et al. CD4OL expressed from the canarypox vector, ALVAC, can boost immunogenicity of HIV-1 canarypox vaccine in mice and enhance the in vitro expansion of viral specific CD8 + Tcell memory responses from HIV-1-infected and HIV-1-uninfected individuals. Vaccine 26, 4062-4072 (2008).

34. Gómez, C.E., Nájera, J.L., Sánchez, R., Jiménez, V. \& Esteban, M. Multimeric soluble CD40 ligand (SCD4OL) efficiently enhances HIV specific cellular immune responses during DNA prime and boost with attenuated poxvirus vectors MVA and NYVAC expressing HIV antigens. Vaccine $\mathbf{2 7}$, 3165-3174 (2009)

35. Lin, F.C., Peng, Y., Jones, L.A., Verardi, P.H. \& Yilma, T.D. Incorporation of CD40 ligand into the envelope of pseudotyped single-cycle simian immunodeficiency viruses enhances immunogenicity. J. Virol. 83, 1216-1227 (2009).

36. Cao, J., Wang, X., Du, Y., Li, Y., Wang, X. \& Jiang, P. CD40 ligand expressed in adenovirus can improve the immunogenicity of the GP3 and GP5 of porcine reproductive and respiratory syndrome virus in swine. Vaccine $\mathbf{2 8}$, 7514-7522 (2010)

37. Hangalapura, B.N. et al. Selective transduction of dendritic cells in human lymph nodes and superior induction of high-avidity melanoma-reactive cytotoxic T cells by a CD40-targeted adenovirus. J. Immunother. 33, 706-715 (2010).

38. Kim, Y.-S. et al. CD40-targeted recombinant adenovirus significantly enhances the efficacy of antitumor vaccines based on dendritic cells and $B$ cells. Hum. Gene Ther. 21, 1697-1706 (2010).

39. Franco, D., Liu, W., Gardiner, D.F., Hahn, B.H. \& Ho, D.D. CD4OLcontaining virus-like particle as a candidate HIV-1 vaccine targeting dendritic cells. J. Acquir. Immune Defic. Syndr. 56, 393-400 (2011).

40. Yao, Q. et al. Immunogenicity and protective efficacy of a DNA vaccine encoding a chimeric protein of avian influenza hemagglutinin subtype $\mathrm{H} 5$ fused to CD154 (CD4OL) in Pekin ducks. Vaccine 28, 8147-8156 (2010).

41. Sánchez Ramos, O. et al. Avian CD154 enhances humoral and cellular immune responses induced by an adenovirus vector-based vaccine in chickens. Comp. Immunol. Microbiol. Infect. Dis. 34, 259-265 (2011).

42. Epstein, S.L. Prior H1N1 influenza infection and susceptibility of Cleveland Family Study participants during the H2N2 pandemic of 1957: an experiment of nature. J. Infect. Dis. 193, 49-53 (2006).

43. Gerhard, W. The role of the antibody response in influenza virus infection. Curr. Top. Microbiol. Immunol. 260, 171-190 (2001).

44. Stanekova, Z. et al. Epitope specificity of anti-HA2 antibodies induced in humans during influenza infection. Influenza Resp. Vir 6, 389-395 (2012).

45. Varečková, E. et al. HA2 glycopolypeptide of influenza A virus and antiviral immunity. Acta Virol 57, 247 V 256 (2013).

46. Carragher, D.M., Kaminski, D.A., Moquin, A., Hartson, L. \& Randall, T.D. A novel role for non-neutralizing antibodies against nucleoprotein in facilitating resistance to influenza virus. J. Immunol. 181, 4168-4176 (2008).

47. Flaman, A.S., Gravel, C., Hashem, A.M., Tocchi, M. \& Li, X. The effect of interferon- $\alpha$ on the expression of cytochrome P450 3A4 in human hepatoma cells. Toxicol. Appl. Pharmacol. 253, 130-136 (2011).

48. Reed, J. \& Muench, H. A simple method of estimating fifty per cent endpoints. Am. J. Epidemiol. 27, 493-497 (1938).

49. Hauser, H., Shen, L., Gu, Q.-L., Krueger, S. \& Chen, S.-Y. Secretory heatshock protein as a dendritic cell-targeting molecule: a new strategy to enhance the potency of genetic vaccines. Gene Ther 11, 924-932 (2004).

50. Hashem, A.M. et al. Subcutaneous immunization with recombinant adenovirus expressing influenza A nucleoprotein protects mice against lethal viral challenge. Hum. Vaccin. Immunother 8, 425-430 (2012).

(1) (2) This work is licensed under the Creative Commons Attribution-NonCommercial-Share Alike 3.0 Unported License. To view a copy of this license, visit http:// creativecommons.org/licenses/by-nc-sa/3.0/ 